\title{
Consistent integration of the constitutive equations of Gurson materials under adiabatic conditions
}

\author{
G. Vadillo, R. Zaera, J. Fernández-Sáez* \\ Department of Continuum Mechanics and Structural Analysis, University Carlos III of Madrid, Avda. de la Universidad, 30, 28911 Leganés, Madrid, Spain
}

\begin{abstract}
In this paper, a consistent integration procedure for the thermoviscoplastic version of the complete Gurson model is proposed. With adiabatic conditions considered and with the use of the backward Euler integration scheme, a numerical algorithm implicit in all vari ables as well as the corresponding algorithmic operator have been developed. The proposed algorithm was implemented in a finite ele ment code and its performance is demonstrated with the numerical simulation of different examples.
\end{abstract}

Keywords: Gurson model; Thermoviscoplasticity; Consistent integration

\section{Introduction}

It is well known that the mechanical properties of an alloy will change under different strain-rate loadings. Thus, an understanding of the constitutive behaviour of metals over a wide range of strain rates is of importance in many advanced processes in engineering, such as metal forming [1], machining [2,3], structures under crashes [4] and highspeed impact on metallic armours $[5,6]$. The strain-rate sensitivity, defined as the amount of change of flow stress because of a change in strain rate, greatly helps to resist instabilities, and thus should be considered in all these processes. Viscoplasticity, as a branch of the theory of solid mechanics, analyses the effect of strain rate in the inelastic properties of the material. A widely used viscoplastic formulation is the overstress model (such as Perzyna [7] and Duvaut-Lions [8]). The main feature of overstress models is that the rate-independent yield function can become larger than zero, allowing excursions of stress states outside of the static yield surface. With the use of the overstress mod-

\footnotetext{
* Corresponding author. Fax: +34916249430.

E mail address: ppfer@ing.uc3m.es (J. Fernández Sáez).
}

els, the consistency condition is not fulfilled and the Kuhn Tucker conditions are not applicable.

A second approach to describe viscoplastic effects is referred to as the consistency model, first proposed by Wang et al. [9] and used by other authors (Ristinmaa and Ottosen [10], Winnicki et al. [11] and Heeres [12]). In the consistency approach, a rate-dependent yield surface is employed and it can expand and shrink not only by softening or hardening effects, but also by softening/hardening rate effects, i.e.,

$f(\boldsymbol{\sigma}, \boldsymbol{\kappa}, \dot{\boldsymbol{\kappa}})=0 \quad$ at $\dot{\lambda}>0$

with $\boldsymbol{\kappa}$ being a vector including all the state variables and $\lambda$ the plastic multiplier. The standard Kuhn Tucker conditions for loading and unloading remain valid when using this formulation. Furthermore, the consistency model yields numerical algorithms with a somewhat higher convergence rate than that derived by the overstress model $[11,13]$.

The above-mentioned processes involving high strain rates are often accompanied by a rise in temperature due to the dissipation of plastic work. This means that the energy-balance equation governing temperature evolution should involve terms arising from a thermomechanical 
coupling. This effect cannot be avoided in most cases in finite deformation problems, especially when the material is heated rapidly and the amount of local heat transfer decreases due to limited thermal diffusion. The thermodynamic process deviates from the isothermal conditions and approaches adiabaticity, leading to large variations in the temperature field. Calculations should then use temperature-variable mechanical properties, and thermal softening of the material should be considered since dynamic plastic instabilities, such as adiabatic shear bands or necking, are commonly triggered by a decrease in the yield limit with increasing temperatures [14 17]. Numerical investigations in thermoviscoplasticity are frequently related to overstress models [18,19]. Recently, Zaera and Fernández-Sáez [20] have extended the consistency model to analyse the thermoviscoplastic behaviour of metals. They have proposed a fully implicit scheme which is easily implemented and inherits the robustness and stability of return mapping algorithms.

The above works do not take into account the micromechanics phenomena responsible for damage and fracture when ductile metals are used. In these cases, processes can be accompanied by major accumulated damage, causing internal deterioration and macroscopic failure. In most ductile metals, the damage mechanisms involve the nucleation of voids at second particles, by decohesion of the particle matrix interface or by particle fracture, the further growth of voids due to the plastic straining of the surrounding matrix and, finally, the coalescence to form a macroscopic crack.

In the continuum-mechanics framework, the most widely used model to describe the aforementioned processes was originally developed by Gurson [21] and further improved by Tvergaard [22,23] and Tvergaard and Needleman [24] (the so-called GTN model). This model predicts the coalescence of voids when a critical volume fraction of them, empirically selected, is reached. Following the plastic-limit-load approach proposed by Thomason [25] a new criterion for void coalescence has been incorporated into the GTN model (called the complete Gurson model [26 28]). This complete Gurson model considers the critical volume fraction of the void as a material- and stress-dependent parameter.

Different authors have applied the Gurson model to dynamic problems, involving inertial and high-strain-rate effects [29 35]. Recently, Betegón et al. [36] included strain-rate effects in the consistency model of Gurson materials and they have proposed an implicit method to integrate the constitutive equations of ductile metallic materials under high strain rates based on the complete Gurson model.

In the above-mentioned works, the thermal effects accompanying the deformation processes at high strain rates are not taken into account. Srikanth and Zabaras [37] proposed a thermoplastic model coupled with ductile damage using the GTN approach to analyse metal forming processes. Thermoviscoplastic versions of the Gurson model, including strain-rate and temperature effects, has been developed by Koppenhoefer and Dodds [38], Eberle et al. [39], Needleman and Tvergaard [40], Tvergaard and Needleman [41], and Hao and Brock [42].

In the present paper, thermal effects are included in the consistency viscoplastic model of void-containing materials modelled by the Gurson constitutive equations, and a consistent integration procedure for the thermoviscoplastic version of the complete Gurson model is proposed. With the consideration of adiabatic conditions and with the use of the backward Euler integration scheme, a numerical algorithm implicit in all variables as well as the corresponding algorithmic operator was developed. The proposed algorithm was implemented in the finite element commercial codes ABAQUS/Standard [43] and ABAQUS/Explicit [44] through the material subroutines UMAT and $V U M A T$, respectively, and its performance is demonstrated with the numerical simulation of different examples.

\section{A themoviscoplastic version of the complete Gurson model}

\subsection{Basic kinematics}

Let $\mathscr{B}_{t} \subset \mathbb{R}^{3}$ define the current configuration at time $t \in \mathbb{R}$ of a continuum body $\mathscr{B}$, and $\mathscr{B}_{0} \subset \mathbb{R}^{3}$ the reference, initial or undeformed configuration at time $t=0$ (considered coincident). Let $X \in \mathscr{B}$ be a particle in the body, $\mathbf{X} \in \mathscr{B}_{0}$ the position of $X$ in the reference configuration $\mathscr{B}_{0}$, and $\mathbf{x} \in \mathscr{B}_{t}$ the position of $X$ in the current configuration $\mathscr{B}_{t}$. The motion of the body is defined by a smooth time-dependent mapping $\varphi_{t}: \mathscr{B}_{0} \rightarrow \mathscr{B}_{t}$, that is $\mathbf{x}=\varphi_{t}(\mathbf{X})$. The deformation gradient $\mathbf{F}$ is a two-point tensor defined by

$\mathbf{F}=\nabla_{\mathbf{X}} \varphi_{t}(\mathbf{X})=\frac{\partial \mathbf{x}}{\partial \mathbf{X}}$

This tensor transforms an infinitesimal material vector $\mathrm{d} \mathbf{X} \in \mathscr{B}_{0}$ into the corresponding spatial vector $\mathrm{d} \mathbf{x} \in \mathscr{B}_{t}$ :

$\mathrm{d} \mathbf{x}=\mathbf{F} \mathrm{d} \mathbf{X}$.

The application of the theorem of polar decomposition to F implies:

$\mathbf{F}=\mathbf{R} \mathbf{U}=\mathbf{V R}$,

$\mathbf{R}$ being the polar orthogonal rotation tensor, $\mathbf{U}$ the material or right stretch tensor and $\mathbf{V}$ the spatial or left stretch tensor. The velocity of a particle $\mathbf{v}_{t}$ at time $t$ is defined consistently as the time derivative of the spatial position $\mathbf{x}$ :

$\mathbf{v}_{t}(\mathbf{x})=\frac{\partial \varphi_{t}^{-1}(\mathbf{x})}{\partial t}$.

The velocity gradient tensor $\mathbf{l}$ is the spatial derivative of $\mathbf{v}_{t}$, which is given by

$\mathbf{l}=\nabla_{\mathbf{x}} \mathbf{v}_{t}=\frac{\partial \mathbf{v}_{t}(\mathbf{x})}{\partial \mathbf{x}}=\dot{\mathbf{F}} \mathbf{F}^{-1}$.

The symmetric and skew-symmetric parts of the latter expression supply two additional rate tensors: the rate of deformation tensor $\mathbf{d}$ and the spin tensor w: 
$\mathbf{d}=\frac{1}{2}\left(\mathbf{l}+\mathbf{l}^{\mathrm{T}}\right)$,

$\mathbf{w}=\frac{1}{2}\left(\mathbf{l}-\mathbf{l}^{\mathrm{T}}\right)$.

\subsection{Decomposition of the deformation gradient and of the} rate of deformation tensor

To separate the recoverable and non-recoverable contributions of the deformation gradient, the Kröner Lee multiplicative split of $\mathbf{F}$ is assumed $[45,46]$ :

$\mathbf{F}=\mathbf{F}^{r} \mathbf{F}^{p}$

$\mathbf{F}^{r}$ represents the reversible thermoelastic deformation of the material:

$\mathbf{F}^{r}=\mathbf{F}^{e} \mathbf{F}^{\theta}$

and $\mathbf{F}^{p}$ represents the inelastic deformation of the material. This decomposition implies the so-called plastic intermediate configuration defined by $\mathbf{F}^{r^{1}}$, which is valid only locally. We may also write the spatial velocity gradient as

$\mathbf{l}=\dot{\mathbf{F}} \mathbf{F}^{-1}=\mathbf{l}^{e}+\mathbf{F}^{e} \mathbf{l}^{\theta} \mathbf{F}^{e^{1}}+\mathbf{F}^{e} \mathbf{l}^{p} \mathbf{F}^{e^{1}}$

$\mathbf{l}^{e}, \mathbf{l}^{\theta}$ and $\mathbf{l}^{p}$ being the elastic, thermal, and plastic spatial velocity gradients defined as

$\mathbf{l}^{e}=\dot{\mathbf{F}}^{e} \mathbf{F}^{e^{1}}, \quad \mathbf{l}^{\theta}=\dot{\mathbf{F}}^{\theta} \mathbf{F}^{\theta^{1}}, \quad \mathbf{l}^{p}=\dot{\mathbf{F}}^{p} \mathbf{F}^{p^{1}}$

while taking into account that thermal deformation is assumed in this work to be purely volumetric, i.e.,

$\mathbf{F}^{\theta}=\left[\operatorname{det}\left(\mathbf{F}^{\theta}\right)\right]^{1 / 3} \mathbf{1}$,

where $\mathbf{1}$ is the unit second-order tensor.

For structural metals used in crashworthiness, manufacturing or ballistic applications, elastic strains (and rates) are commonly very small compared to unity or to plastic strains (and rates). With this restriction, we get the following approximations:

$\mathbf{F}^{e}=\mathbf{U}^{e}=\mathbf{V}^{e}=\mathbf{1}+\mathrm{O}\left(\varepsilon^{e}\right)$.

From this, Eq. (11) leads to

$\mathbf{l}=\mathbf{l}^{e}+\mathbf{l}^{\theta}+\mathbf{l}^{p}$

and its symmetric part to the additive decomposition of the rate of deformation tensor $\mathbf{d}$, generally assumed for hypoelastic plastic materials $[47,48]$ :

$\mathbf{d}=\mathbf{d}^{e}+\mathbf{d}^{\theta}+\mathbf{d}^{p}$.

\subsection{Constitutive equations}

The elastic strain is provided by an objective time derivative of the generalized Hooke's law:

$\boldsymbol{\sigma}^{\nabla}=\mathbf{C}: \mathbf{d}^{e}=\mathbf{C}:\left(\mathbf{d}-\mathbf{d}^{\theta}-\mathbf{d}^{p}\right)$,

where $\boldsymbol{\sigma}$ is the Cauchy stress and $\mathbf{C}$ is the Hooke stress strain tensor given by
$\mathbf{C}=2 G \mathbf{I}^{\mathrm{dev}}+K \mathbf{1} \otimes \mathbf{1}$

with $G$ and $K$ being elastic constants:

$G=\frac{E}{2(1+v)}$,

$K=\frac{E}{3(1-2 v)}$,

and $\mathbf{I}^{\text {dev }}$ the unit deviatoric fourth-order tensor given by

$\left(\mathbf{I}^{\mathrm{dev}}\right)_{i j k l}=\frac{1}{2}\left(\delta_{i k} \delta_{j l}+\delta_{i l} \delta_{j k}\right)-\frac{1}{3} \delta_{i j} \delta_{k l}$

The yield function $\Phi$ considered for the porous solid is the one originally proposed by Gurson [21] and modified by Tvergaard [22,23] and Tvergaard and Needleman [24]. Function $\Phi=0$ defines the boundary of the elastic domain:

$$
\begin{aligned}
\Phi\left(q, p, \sigma, f^{*}\right)= & \frac{q^{2}}{\sigma^{2}}+2 q_{1} f^{*} \cosh \left(\frac{3 q_{2} p}{2 \sigma}\right) \\
& -1-\left(q_{1} f^{*}\right)^{2}=0,
\end{aligned}
$$

where $q=\sqrt{\frac{3}{2}} \mathbf{s}: \mathbf{s}$ is the macroscopic effective stress, $p=-\frac{1}{3} \boldsymbol{\sigma}: \mathbf{1}$ is the hydrostatic pressure and $\mathbf{s}=\boldsymbol{\sigma}+p \mathbf{1}$ the stress deviator. Also, $\sigma=\sigma\left(\varepsilon^{p}, \dot{\varepsilon}^{p}, \theta\right)$ is the flow stress of the matrix material, which depends on the equivalent plastic strain $\varepsilon^{p}$, the equivalent plastic strain rate $\dot{\varepsilon}^{p}\left(\varepsilon^{p}=\int_{0}^{t} \dot{\varepsilon}^{p}(\tau) \mathrm{d} \tau\right)$, and the temperature $\theta ; q_{1}$ and $q_{2}$ are fitting parameters [22,23], and, finally, $f^{*}$ is the modified void-volume fraction, introduced by Tvergaard and Needleman [24] to improve the prediction of final failure due to void coalescence, given by

$f^{*}= \begin{cases}f & \text { if } f<f_{\mathrm{c}}, \\ f_{\mathrm{c}}+\frac{\left(f_{\mathrm{u}}-f_{\mathrm{c}}\right)}{\left(f_{\mathrm{F}}-f_{\mathrm{c}}\right)}\left(f-f_{\mathrm{c}}\right) & \text { if } f_{\mathrm{c}} \leqslant f \leqslant f_{\mathrm{F}}, \\ f_{\mathrm{u}} & \text { if } f>f_{\mathrm{F}},\end{cases}$

where $f$ is the volume fraction of voids (porosity), $f_{\mathrm{c}}$ the critical void-volume fraction at which voids coalesce, $f_{\mathrm{F}}$ the void-volume fraction at final failure of the material, and $f_{\mathrm{u}}=1 / q_{1}$ the ultimate void-volume fraction. The value of the critical void-volume fraction where coalescence begins, $f_{\mathrm{c}}$, is considered here not as a constant material value, as in the original paper of Gurson [21], but as a field quantity that can be determined by the Thomason plastic limit load criterion [25]. The main idea of Thomason model is that void coalescence coincides with the plastic limit load condition for localized deformation of intervoid matrix. Zhang [26] and Zhang et al. [27] modified the plastic-limit-load of Thomason, assuming that the voids grow spherically, without shape changes. According to this, in a general three-dimensional problem, void coalescence starts if $[26,27]$ :

$\frac{\sigma_{1}}{\sigma}=\left(\gamma\left(\frac{1}{r}-1\right)^{2}+\frac{\beta}{\sqrt{ } r}\right)\left(1-\pi r^{2}\right)$,

but it will not occur if $\frac{\sigma_{1}}{\sigma}$ does not reach this value. Then, $f_{\mathrm{c}}$ is taken as the current value of $f$ at the specific material point where condition (23) is fulfilled. In Eq. (23), $\sigma_{1}$ is 
the maximum principal stress and $r$ is the void-space ratio defined by

$r=\frac{\sqrt[3]{\frac{3 f}{4 \pi}} \mathrm{e}^{\varepsilon_{1}+\varepsilon_{2}+\varepsilon_{3}}}{\frac{\sqrt{\mathrm{e}^{\varepsilon_{2}+\varepsilon_{3}}}}{2}}$

with $\varepsilon_{1}, \varepsilon_{2}$ and $\varepsilon_{3}$ the principal strains, $\gamma=0.1$ and $\beta=1.2$ constants fitted by Thomason [25] for the non-hardening materials. Pardoen and Hutchinson [28] proposed a dependence of $\gamma$ with the coefficient $N$ in the Ramberg Osgood $\left(\sigma=\sigma_{0}\left(\varepsilon^{p} / \varepsilon_{0}\right)^{N}\right)$ strain hardening law. From finite element cell model analysis, where void shape, hardening, and initial void-volume fraction were varied, they found that the following relations fit better, in average sense, to all the cases they studied:

$\gamma=0.1+0.217 N+4.83 N^{2}, \quad \beta=1.24$.

If voids are assumed to be always spherical (complete Gurson model), Zhang et al. [27] found that it is more accurate to consider:

$\gamma=0.12+1.68 N, \quad \beta=1.2$.

The complete Gurson model was proposed by Zhang [26], Zhang et al. [27] and Pardoen and Hutchinson [28] using both the modified Gurson and the Thomason criterion. Recently, Betegón et al. [36] have extended this model to dynamic conditions.

The average void-volume fraction changes due partly to the growth of existing voids and partly to the nucleation of new ones:

$\dot{f}=\dot{f}_{\text {growth }}+\dot{f}_{\text {nucleation }}$.

Assuming that elastic strains are negligible, void growth is due to cavity expansion:

$\dot{f}_{\text {growth }}=(1-f) \mathbf{d}^{p}: \mathbf{1}$.

The void nucleation model used here was proposed by Needleman and Rice [49] and it considers that void nucleation is controlled by both plastic strain and stress mechanisms, i.e.,

$\dot{f}_{\text {nucleation }}=A \dot{\varepsilon}^{p}+B(\dot{\sigma}-\dot{p})$.

A material with void nucleation controlled by the plastic strain can be modelled using Eq. (29) by taking $A>0$ and $B \quad 0$. Chu and Needleman [50] proposed a normal distribution for nucleation with the following expression for $A$ and $B$ :

$A=\frac{f_{\mathrm{N}}}{s_{\mathrm{N}} \sqrt{ } 2 \pi} \exp \left[-\frac{1}{2}\left(\frac{\varepsilon-\varepsilon_{\mathrm{N}}}{s_{\mathrm{N}}}\right)^{2}\right], \quad B=0$,

$\varepsilon_{N}$ being the mean strain for nucleation, $s_{\mathrm{N}}$ the corresponding standard deviation, and $f_{\mathrm{N}}$ the volume fraction of nucleating particles.

The plastic rate of deformation is given by the associated flow rule: $\mathbf{d}^{p}=\dot{\lambda} \frac{\partial \Phi}{\partial \boldsymbol{\sigma}}$

and separating into its deviatoric and hydrostatic component:

$\mathbf{d}^{p}=\dot{\lambda}\left(-\frac{1}{3} \frac{\partial \Phi}{\partial p} \mathbf{1}+\frac{\partial \Phi}{\partial q} \mathbf{n}\right)$

with $\mathbf{n}$ being the unit vector in the deviatoric stress space:

$\mathbf{n}=\frac{3}{2} \frac{\mathbf{s}}{q}$.

The thermal rate of deformation tensor, assumed to be isotropic, is written as

$\mathbf{d}^{\theta}=\alpha \dot{\theta} \mathbf{1}$

with $\alpha$ being the coefficient of thermal expansion. Under assumed adiabatic conditions (no heat flux) and plastic work as the only volumetric heat source, the balance of energy gives:

$\rho c_{p} \dot{\theta}=\chi \boldsymbol{\sigma}: \mathbf{d}^{p}$,

where $\rho$ is the actual density, $c_{p}$ the specific heat and $\chi$ the Quinney Taylor coefficient. According to the principle of mass conservation, density is expressed as

$\rho=\frac{\rho_{0}}{\operatorname{det}(\mathbf{F})}$.

Under the assumption that the rate of equivalent plastic work in the matrix material equals the macroscopic rate of plastic work, it follows that:

$\boldsymbol{\sigma}: \mathbf{d}^{p}=(1-f) \sigma \dot{\varepsilon}^{p}$.

The formulation of the model is completed by introducing the Kuhn Tucker loading/unloading complementary conditions:

$\lambda \geqslant 0, \quad \Phi \leqslant 0, \quad \lambda \Phi=0$

and the consistency condition

$\lambda \dot{\Phi}=0$.

\section{Consistent integration procedure}

To integrate the above set of nonlinear rate equations, incremental objectivity is achieved by rewriting them in a neutralized configuration [51 53]. To formalize this approach, being $\varpi$ a spatial skew-symmetric tensor, we may generate a group of rotations $\mathfrak{R}$ such that:

$\dot{\mathfrak{R}}=\boldsymbol{\varpi} \mathfrak{R}, \quad \mathfrak{R}_{\left(\begin{array}{l}t \\ 0\end{array}\right)}=\mathbf{1}$

with

$\varpi=-\boldsymbol{\varpi}^{\mathrm{T}}$

and

$\mathfrak{R}^{-1}=\mathfrak{R}^{\mathrm{T}}$. 
Typical choices of $\boldsymbol{\varpi}$ include the spin tensor $\mathbf{w}$ and the tensor $\boldsymbol{\Omega}$ defined as

$\boldsymbol{\Omega}=\dot{\mathbf{R}} \mathbf{R}^{\mathrm{T}}$,

where $\mathbf{R}$ is the polar rotation tensor. The Cauchy stress tensor and the rate of deformation tensor are rotated as

$\boldsymbol{\sigma}_{\mathfrak{R}}=\mathfrak{R}^{\mathrm{T}} \boldsymbol{\sigma} \mathfrak{R}, \quad \mathbf{d}_{\mathfrak{R}}=\mathfrak{R}^{\mathrm{T}} \mathbf{d} \mathfrak{R}$.

Time differentiation of the rotated Cauchy stress leads to:

$\dot{\boldsymbol{\sigma}}_{\mathfrak{R}}=\mathfrak{R}^{\mathrm{T}}(\dot{\boldsymbol{\sigma}}+\boldsymbol{\sigma} \boldsymbol{\varpi}-\boldsymbol{\varpi} \boldsymbol{\sigma}) \mathfrak{R}=\mathfrak{R}^{\mathrm{T}} \boldsymbol{\sigma}^{\nabla} \mathfrak{R}$,

where $\boldsymbol{\sigma}^{\nabla}$ coincides with the Jaumann stress rate if $\boldsymbol{\varpi}=\mathbf{w}$. The Hughes Winget algorithm [54] is used to compute $\mathfrak{R}$ in this case with an approximated formula valid for small increments. If $\boldsymbol{\sigma}$ is chosen to be equal to $\boldsymbol{\Omega}$ (and then $\mathfrak{R}=\mathbf{R}), \boldsymbol{\sigma}^{\nabla}$ coincides with the Green Naghdi McInnis stress rate. Thus, a complicated objective stress rate can be computed as a simple time derivative. Taking advantage of the orthogonality of $\mathfrak{R}$, the symmetry of the Cauchy stress and rate of deformation tensors and the isotropy of the elastic tensor $\left(\mathbf{C}_{\mathfrak{R}}=\mathbf{C}\right)$, we find that the equations defined above, in which tensors are involved, are form-identical in the corotational configuration but with spatial variables now replaced by rotated variables:

$\dot{\boldsymbol{\sigma}}_{\mathfrak{R}}=\mathbf{C}:\left(\mathbf{d}_{\mathfrak{R}}-\mathbf{d}_{\mathfrak{R}}^{\theta}-\mathbf{d}_{\mathfrak{R}}^{p}\right)$,

$\mathbf{d}_{\mathfrak{R}}^{\theta}=\alpha \dot{\theta} \mathbf{1}$,

$\mathbf{d}_{\Re}^{p}=\dot{\lambda} \frac{\partial \Phi}{\partial \boldsymbol{\sigma}_{\mathfrak{R}}}=\dot{\lambda}\left(-\frac{1}{3} \frac{\partial \Phi}{\partial p} \mathbf{1}+\frac{\partial \Phi}{\partial q} \mathbf{n}_{\Re}\right)$,

$p=-\frac{1}{3} \boldsymbol{\sigma}_{\Re}: \mathbf{1}, \quad q=\sqrt{\frac{3}{2}} \mathbf{S}_{\mathfrak{R}}: \mathbf{S}_{\mathfrak{R}}$,

$\boldsymbol{\sigma}_{\mathfrak{R}}=\mathbf{s}_{\mathfrak{R}}-p \mathbf{1}, \quad \mathbf{n}_{\mathfrak{R}}=\frac{3}{2} \frac{\mathbf{s}_{\mathfrak{R}}}{q}$,

$\rho c_{p} \dot{\theta}=\chi \boldsymbol{\sigma}_{\mathfrak{R}}: \mathbf{d}_{\mathfrak{R}}^{p}$,

$\boldsymbol{\sigma}_{\mathfrak{R}}: \mathbf{d}_{\Re}^{p}=(1-f) \sigma \dot{\varepsilon}^{p}$,

$\dot{f}=(1-f) \mathbf{d}_{\Re}^{p}: \mathbf{1}+A \dot{\varepsilon}^{p}+B(\dot{\sigma}-\dot{p})$,

and all the constitutive equations of the model involving only scalars remain changeless.

\subsection{The solving algorithm}

Within the finite element method, the integration process is local in space, that is, it occurs at quadrature points of the finite elements. The incremental integration of the constitutive model is regarded as a strain-driven process in which the total strain tensor increment at each quadrature point is given at a certain time and both the stress and the state variables should be updated. The re-writing of the above equations in the corotational configuration guarantees incrementally objective stress update [51 53].

Within the frame of the neutralized configuration, the classical return mapping algorithm is proposed to solve the above equations [51,52]. The return is performed at time $n+1$ with the corresponding updated rotated stress:

$\boldsymbol{\sigma}_{\mathfrak{R}_{n+1}}=\boldsymbol{\sigma}_{\mathfrak{R} n+1}^{\mathrm{trial}}+\Delta \boldsymbol{\sigma}_{\mathfrak{R}}^{\theta}+\Delta \boldsymbol{\sigma}_{\mathfrak{R}}^{\mathrm{ret}}$,

where rotated trial stress is given by

$\boldsymbol{\sigma}_{\mathfrak{R}_{n+1}}^{\text {trial }}=\boldsymbol{\sigma}_{\mathfrak{R}_{n}}+\mathbf{C}:\left(\Delta \boldsymbol{\varepsilon}_{\mathfrak{R}}\right)$

with $\boldsymbol{\sigma}_{\Re_{n}}$ being the rotated stress at time $n$ :

$\boldsymbol{\sigma}_{\mathfrak{R}_{n}}=\mathfrak{R}_{n}^{\mathrm{T}} \boldsymbol{\sigma}_{n} \mathfrak{R}_{n}$,

and $\Delta \varepsilon_{\mathfrak{R}}$ the increment of total deformation in the neutralized frame, which could be determined by an objective approximation of the rate of deformation tensor $\mathbf{d}_{n+1 / 2}$ calculated by the midpoint rule (see [51,52]):

$\Delta \boldsymbol{\varepsilon}_{\mathfrak{R}}=\Delta t \mathbf{d}_{\mathfrak{R} n+1 / 2}=\Delta t \mathfrak{R}_{n+1 / 2}^{\mathrm{T}} \mathbf{d}_{n+1 / 2} \mathfrak{R}_{n+1 / 2}$.

A fully implicit backward Euler scheme is used to correct the trial stress:

$\Delta \boldsymbol{\sigma}_{\Re}^{\theta}=-3 K \alpha \Delta \theta \mathbf{1}$,

$\Delta \boldsymbol{\sigma}_{\mathfrak{R}}^{\mathrm{ret}}=-\mathbf{C}: \Delta \lambda\left[-\frac{1}{3}\left(\frac{\partial \Phi}{\partial p}\right)_{n+1} \mathbf{1}+\left(\frac{\partial \Phi}{\partial q}\right)_{n+1} \mathbf{n}_{\mathfrak{R}_{n+1}}\right]$.

Using the variables introduced by Aravas [55]:

$\Delta \varepsilon_{\mathrm{p}}=-\Delta \lambda\left(\frac{\partial \Phi}{\partial p}\right)_{n+1}, \quad \Delta \varepsilon_{\mathrm{q}}=\Delta \lambda\left(\frac{\partial \Phi}{\partial q}\right)_{n+1}$.

Eq. (58) can be written as

$\Delta \boldsymbol{\sigma}_{\mathfrak{R}}^{\mathrm{ret}}=-K \Delta \varepsilon_{\mathrm{p}} \mathbf{1}-2 G \Delta \varepsilon_{\mathrm{q}} \mathbf{n}_{\mathfrak{R}_{n+1}}$.

The updated deviatoric stress is then given by

$\mathbf{S}_{\mathfrak{R}_{n+1}}=\mathbf{s}_{\mathfrak{R}_{n+1}}^{\text {trial }}-3 G \Delta \varepsilon_{\mathrm{q}} \frac{\mathbf{S}_{\Re_{n+1}}}{q_{n+1}}$.

This last equation clearly shows the proportionality between $\mathbf{S}_{\mathfrak{R}_{n+1}}$ and $\mathbf{s}_{\mathfrak{R}_{n+1}}^{\text {trial }}$, which could be equally stated as

$\frac{\mathbf{S}_{\mathfrak{R}_{n+1}}}{q_{n+1}}=\frac{\mathbf{S}_{\mathfrak{R}_{n+1}}^{\text {trial }}}{q_{n+1}^{\text {trial }}}$

or

$\mathbf{n}_{\Re_{n+1}}=\mathbf{n}_{\mathfrak{R}_{n+1}}^{\text {trial }}$,

where $q_{n+1}^{\text {trial }}=\sqrt{\frac{3}{2}} \mathbf{S}_{\mathfrak{R}_{n+1}}^{\text {trial }}: \mathbf{s}_{\mathfrak{R}_{n+1}}^{\text {trial }}$. This equation shows that the value of the unit vector $\mathbf{n}_{\mathfrak{R}_{n+1}}$ can be simply determined from the value of the rotated elastic deviatoric stress predictor $\mathbf{S}_{\mathfrak{R}_{n+1}}^{\text {trial }}$.

Separating Eq. (53) into deviatoric and hydrostatic components according to (60), the resulting equations of the updated rotated stress are

$$
\begin{aligned}
& p_{n+1}=p_{n+1}^{\text {trial }}+K \Delta \varepsilon_{\mathrm{p}}+3 K \alpha \Delta \theta, \\
& q_{n+1}=q_{n+1}^{\text {trial }}-3 G \Delta \varepsilon_{\mathrm{q}} .
\end{aligned}
$$

After some algebra, the equations in (59) can be written as

$\Delta \varepsilon_{\mathrm{p}}\left(\frac{\partial \Phi}{\partial q}\right)_{n+1}+\Delta \varepsilon_{\mathrm{q}}\left(\frac{\partial \Phi}{\partial p}\right)_{n+1}=0$. 
The evolution of the four state variables $\theta, f, \varepsilon^{p}, \dot{\varepsilon}^{p}$ are given

$\Delta \theta=\chi\left(q \Delta \varepsilon_{\mathrm{q}}-p \Delta \varepsilon_{\mathrm{p}}\right) /\left(\rho c_{p}\right)$,

$\Delta f=(1-f) \Delta \varepsilon_{\mathrm{p}}+A \Delta \overline{\varepsilon^{p}}+B(\Delta \sigma-\Delta p)$,

$\Delta \varepsilon^{p}=\left(q \Delta \varepsilon_{\mathrm{q}}-p \Delta \varepsilon_{\mathrm{p}}\right) /((1-f) \sigma)$,

$\Delta \dot{\varepsilon}^{p}=\frac{\Delta \varepsilon^{p}}{\Delta t}$

with variables evaluated in $n+1$ (omitted for simplicity) and $\Delta t$ known. $[55]$

This set of four evolution equations can be written as

$\Delta \mathbf{H}=\mathbf{h}\left(\Delta \varepsilon_{\mathrm{p}}, \Delta \varepsilon_{\mathrm{q}}, p, q, \mathbf{H}\right)$

with $\mathbf{H}$ being a vector containing $\theta, f, \varepsilon^{p}$ and $\dot{\varepsilon}^{p}$ variables.

The problem of integration is reduced to the solution of the following set of eight non-linear equations:

$\Delta \varepsilon_{\mathrm{p}} \frac{\partial \Phi}{\partial q}+\Delta \varepsilon_{\mathrm{q}} \frac{\partial \Phi}{\partial p}=0$

$\Phi\left(p, q, H^{\alpha}\right)=0$,

$p=p^{\text {trial }}+K \Delta \varepsilon_{\mathrm{p}}+3 K \alpha \Delta \theta$,

$q=q^{\text {trial }}-3 G \Delta \varepsilon_{\mathrm{q}}$,

$\Delta \mathbf{H}=\mathbf{h}\left(\Delta \varepsilon_{\mathrm{p}}, \Delta \varepsilon_{\mathrm{q}}, p, q, \mathbf{H}\right)$,

solved using a standard method (Newton Raphson).

The updated rotated stress is equal to:

$\boldsymbol{\sigma}_{\mathfrak{R}_{n+1}}=\frac{2}{3} q_{n+1} \mathbf{n}_{n+1}^{\text {trial }}-p_{n+1} \mathbf{1}$

and $\boldsymbol{\sigma}_{n+1}$ is obtained by the updated rotated stress being pushed to the spatial configuration by $\mathfrak{R}_{n+1}$ :

$\boldsymbol{\sigma}_{n+1}=\mathfrak{R}_{n+1} \boldsymbol{\sigma}_{\mathfrak{R}_{n+1}} \mathfrak{R}_{n+1}^{\mathrm{T}}$.

\section{Algorithmic tangent operator}

For infinitesimal strain problems Simo and Taylor [56] proposed the use of a consistent tangent operator that is, in general, different from the continuum tangent moduli and dependent on the algorithm used for the integration of the constitutive equations by enforcement of the consistency condition at the end of the step $t_{n+1}$. For Gurson materials, an explicit expression of the tangent operator consistent with the Euler backward algorithm has been given by Aravas [55] and Zhang [57]. The use of this kind of tangent operator preserves the quadratic rate of asymptotic convergence of iterative solution schemes based on the Newton method. For the finite transformation regime, we followed an algorithmic strategy similar to that proposed by Lin and Brocks [58], Lin et al. [59] and Doghri [52]. First, the rotated tangent operator, $\mathbf{J}_{\mathfrak{R}}$, is calculated in the neutralized frame and afterwards, is rotated with $\mathfrak{R}_{n+1}$.
To determine $\mathbf{J}_{\mathfrak{R}}$, we proceed as follows:

$\mathbf{J}_{\mathfrak{R}}=\left(\frac{\partial \boldsymbol{\sigma}_{\mathfrak{R}}}{\partial \boldsymbol{\varepsilon}_{\mathfrak{R}}}\right)_{n+1}=\left(\frac{\partial \Delta \boldsymbol{\sigma}_{\mathfrak{R}}}{\partial \Delta \boldsymbol{\varepsilon}_{\mathfrak{R}}}\right)_{n+1}$.

From Eqs. (53) and (54):

$\sigma_{\mathfrak{R}_{n+1}}=\sigma_{\mathfrak{R}_{n}}+\mathbf{C}:\left(\Delta \boldsymbol{\varepsilon}_{\mathfrak{R}}-\Delta \varepsilon_{\mathfrak{R}}^{\theta}-\Delta \varepsilon_{\mathfrak{R}}^{\text {ret }}\right)$.

Deriving the discretized Eq. (73), and considering separately the hydrostatic and deviatoric components, we get:

$$
\begin{aligned}
\delta \Delta \boldsymbol{\sigma}_{\mathfrak{R}}= & \mathbf{C}: \delta \Delta \boldsymbol{\varepsilon}_{\mathfrak{R}}-\mathbf{C}: \delta \Delta \boldsymbol{\varepsilon}_{\mathfrak{R}}^{\theta}-\mathbf{C}: \delta \Delta \boldsymbol{\varepsilon}_{\mathfrak{R}}^{\mathrm{ret}} \\
= & \left(2 G \mathbf{I}^{\mathrm{dev}}+K \mathbf{1} \otimes \mathbf{1}\right): \delta \boldsymbol{\varepsilon}_{\mathfrak{R}}-K \delta \Delta \varepsilon_{\mathrm{p}} \mathbf{1} \\
& -2 G \delta \Delta \varepsilon_{\mathrm{q}} \mathbf{n}_{\mathfrak{R}}-2 G \Delta \varepsilon_{\mathrm{q}} \delta \mathbf{n}_{\mathfrak{R}}-3 K \alpha \delta \theta \mathbf{1}
\end{aligned}
$$

where $n+1$ is omitted for simplicity.

From Eqs. (64) and (65):

$$
\begin{aligned}
& \delta p=\delta p^{\text {trial }}+K \delta \Delta \varepsilon_{\mathrm{p}}+3 K \alpha \delta \Delta \theta \\
& \delta q=\delta q^{\text {trial }}-3 G \delta \Delta \varepsilon_{\mathrm{q}}
\end{aligned}
$$

Deriving the plastic flow (66), yield (22), and the evolution of the four-state variables (68), and after some algorithmic manipulations, the following set of equations results:

Table 1

Thermal, elastic, and viscoplastic parameters of the materials considered

\begin{tabular}{llll}
\hline & $\begin{array}{l}\text { Material A } \\
(4340 \text { Steel })\end{array}$ & $\begin{array}{l}\text { Material B } \\
\text { (Armco Iron) }\end{array}$ & $\begin{array}{l}\text { Material C } \\
\text { (Electronic Copper) }\end{array}$ \\
\hline$E(\mathrm{MPa})$ & 208,000 & 209,000 & 124,000 \\
$v$ & 0.3 & 0.3 & 0.3 \\
$\rho_{0}\left(\mathrm{~kg} / \mathrm{m}^{3}\right)$ & 7830 & 7890 & 8960 \\
$K_{1}(\mathrm{MPa})$ & 792 & 175 & 90 \\
$K_{2}(\mathrm{MPa})$ & 510 & 380 & 292 \\
$n$ & 0.26 & 0.23 & 0.31 \\
$K_{3}$ & 0.014 & 0.06 & 0.025 \\
$\overline{\bar{\varepsilon}}_{0}\left(\mathrm{~s}^{1}\right)$ & 1 & 1 & 1 \\
$m$ & 1.03 & 0.55 & 1.09 \\
$\theta_{0}(\mathrm{~K})$ & 298 & 294 & 298 \\
$\theta_{\mathrm{m}}\left(\mathrm{K}^{1}\right)$ & 1793 & 1811 & 1331 \\
$\chi$ & 0.9 & 0.9 & 0.9 \\
$c_{p}\left(\mathrm{~m}^{2} \mathrm{~K}^{1} \mathrm{~s}^{2}\right)$ & 477 & 452 & 383 \\
$\alpha\left(\mathrm{K}^{1}\right)$ & $1.2 \times 10^{5}$ & $1.2 \times 10^{5}$ & $1.7 \times 10^{5}$ \\
\hline
\end{tabular}

Table 2

Parameters of the Gurson model for the materials considered

\begin{tabular}{llll}
\hline & $\begin{array}{l}\text { Material A } \\
(4340 \text { Steel) }\end{array}$ & $\begin{array}{l}\text { Material B } \\
\text { (Armco Iron) }\end{array}$ & $\begin{array}{l}\text { Material C } \\
\text { (Electronic Copper) }\end{array}$ \\
\hline$q_{1}$ & 1.5 & 1.25 & 1.5 \\
$q_{2}$ & 1.0 & 0.95 & 1.0 \\
$f_{0}$ & 0.004 & 0.0004 & 0.001 \\
$f_{\mathrm{N}}$ & 0.1 & 0.005 & 0.1 \\
$s_{\mathrm{N}}$ & 0.3 & 0.025 & 0.3 \\
$\varepsilon_{\mathrm{n}}$ & 0.1 & 0.05 & 0.1 \\
$f_{\mathrm{F}}$ & 0.2025 & 0.06 & 0.025 \\
\hline
\end{tabular}


$\delta \Delta \varepsilon_{\mathrm{p}}=C_{11} \delta p^{\text {trial }}+C_{12} \delta q^{\text {trial }}$

$\delta \Delta \varepsilon_{\mathrm{q}}=C_{21} \delta p^{\text {trial }}+C_{22} \delta q^{\text {trial }}$

$\delta \mathbf{H}=C_{k 1} \delta p^{\text {trial }}+C_{k 2} \delta q^{\text {trial }}, \quad k=3,4,5,6$

with all $C_{k j}$ coefficients known.
(76) Concretely, the derivation of $\theta$ can be written as

$\delta \theta=C_{31} \delta p^{\text {trial }}+C_{32} \delta q^{\text {trial }}$

bearing in mind that:
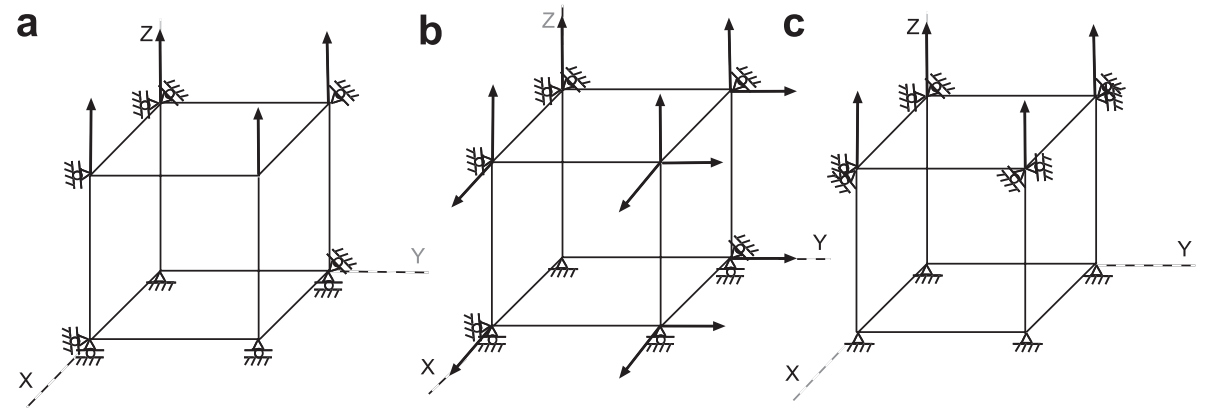

Fig. 1. Single element test cases and boundary conditions: (a) uniaxial extension, (b) volumetric expansion and (c) constrained uniaxial extension.
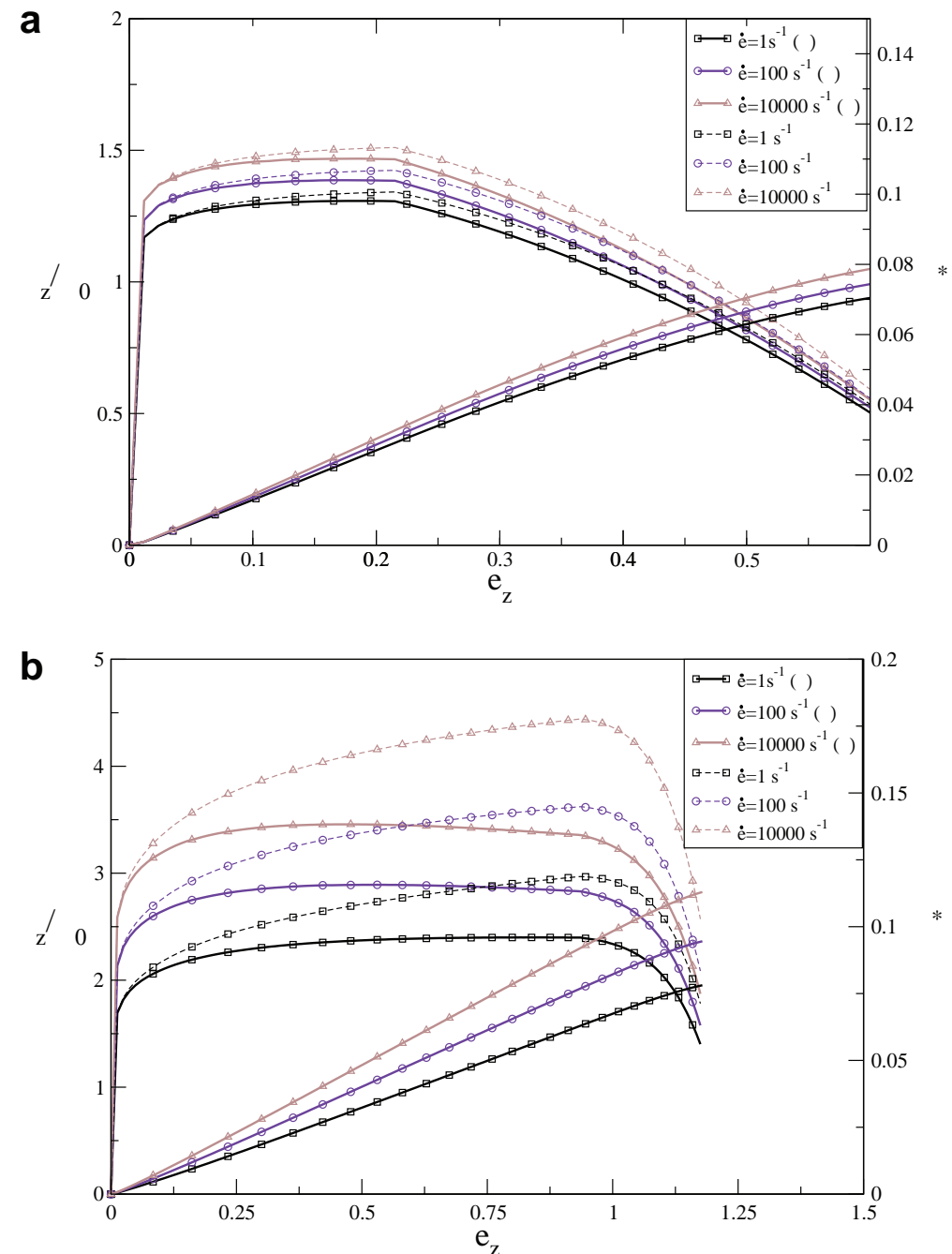

Fig. 2. Axial stress and homologous temperature as a function of axial strain. Uniaxial extension. (a) Material A. (b) Material B. $(\theta)$ indicates consideration of thermal effects. 
$\delta p^{\text {trial }}=-K\left(\delta \boldsymbol{\varepsilon}_{\Re}\right)_{k k}$,

$\delta q^{\text {trial }}=\frac{3}{2 q^{\text {trial }}} \mathbf{s}_{\mathfrak{R}}^{\text {trial }}: \delta \mathbf{s}_{\mathfrak{R}}^{\text {trial }}$,

$\delta \mathbf{s}_{\mathfrak{R}}^{\text {trial }}=2 G\left(\delta \boldsymbol{\varepsilon}_{\mathfrak{R}}-\frac{1}{3}\left(\delta \boldsymbol{\varepsilon}_{\mathfrak{R}}\right)_{k k} \mathbf{1}\right)$,

$\mathbf{n}_{\mathfrak{R}}=\mathbf{n}_{\mathfrak{R}}^{\text {trial }}=\frac{3}{2 q^{\text {trial }}} \mathbf{s}_{\mathfrak{R}}^{\text {trial }}$

$\delta \mathbf{n}_{\mathfrak{R}}=\delta \mathbf{n}_{\mathfrak{R}}^{\text {trial }}=\frac{3}{2 q^{\text {trial }}} \delta \mathbf{s}_{\mathfrak{R}}^{\text {trial }}-\frac{3}{\left(2 q^{\text {trial }}\right)^{2}} \delta q^{\text {trial }} \mathbf{s}_{\mathfrak{R}}^{\text {trial }}$.

From Eqs. (74) and (79), the rotated tangent operator can be written as

$$
\begin{aligned}
\mathbf{J}_{\mathfrak{R}}= & K\left(1+K C_{11}+3 \alpha K C_{31}\right) \mathbf{1} \otimes \mathbf{1}+\left(2 G-6 \frac{G^{2} \Delta \varepsilon_{q}}{q^{\text {trial }}}\right) \mathbf{I}^{\text {dev }} \\
& +\frac{3 K G}{q^{\text {trial }}}\left(C_{12}-3 \alpha C_{32}\right) \mathbf{1} \otimes \mathbf{s}_{\mathfrak{R}}^{\text {trial }}-\frac{3 K G}{q^{\text {trial }}} C_{21} \mathbf{s}_{\mathfrak{R}}^{\text {trial }} \otimes \mathbf{1} \\
& +9 \frac{G^{2}}{\left(q^{\text {trial }}\right)^{2}}\left(-C_{22}+\frac{\Delta \varepsilon_{q}}{q^{\text {trial }}}\right) \mathbf{s}_{\mathfrak{R}}^{\text {trial }} \otimes \mathbf{s}_{\mathfrak{R}}^{\text {trial }}
\end{aligned}
$$

It should be noted, that there is no matrix inversion involved in this process, and the symmetry of this operator is also not preserved.

Since the incremental constitutive equations were derived on the corotational basis, $\mathbf{J}_{\mathfrak{R}}$ corresponds to this frame and it should be pushed forward to obtain the tangent operator by using the rotation matrix $\mathfrak{R}_{n+1}$,

$(\mathbf{J})_{i j k l}=\left(\mathfrak{R}_{n+1}\right)_{i I}\left(\mathfrak{R}_{n+1}\right)_{j J}\left(\mathfrak{R}_{n+1}\right)_{k K}\left(\mathfrak{R}_{n+1}\right)_{l L}\left(\mathbf{J}_{\mathfrak{R}}\right)_{I J K L}$.

This leads to a tensor $\mathbf{J}$ with a mathematical structure identical to $\mathbf{J}_{\mathfrak{R}}$ :

$$
\begin{aligned}
\mathbf{J}= & K\left(1+K C_{11}+3 \alpha K C_{31}\right) \mathbf{1} \otimes \mathbf{1}+\left(2 G-6 \frac{G^{2} \Delta \varepsilon_{q}}{q^{\text {trial }}}\right) \mathbf{I}^{\mathrm{dev}} \\
& +\frac{3 K G}{q^{\text {trial }}}\left(C_{12}-3 \alpha C_{32}\right) \mathbf{1} \otimes \mathbf{s}^{\text {trial }}-\frac{3 K G}{q^{\text {trial }}} C_{21} \mathbf{s}^{\text {trial }} \otimes \mathbf{1} \\
& +9 \frac{G^{2}}{\left(q^{\text {trial }}\right)^{2}}\left(-C_{22}+\frac{\Delta \varepsilon_{q}}{q^{\text {trial }}}\right) \mathbf{s}^{\text {trial }} \otimes \mathbf{s}^{\text {trial }}
\end{aligned}
$$
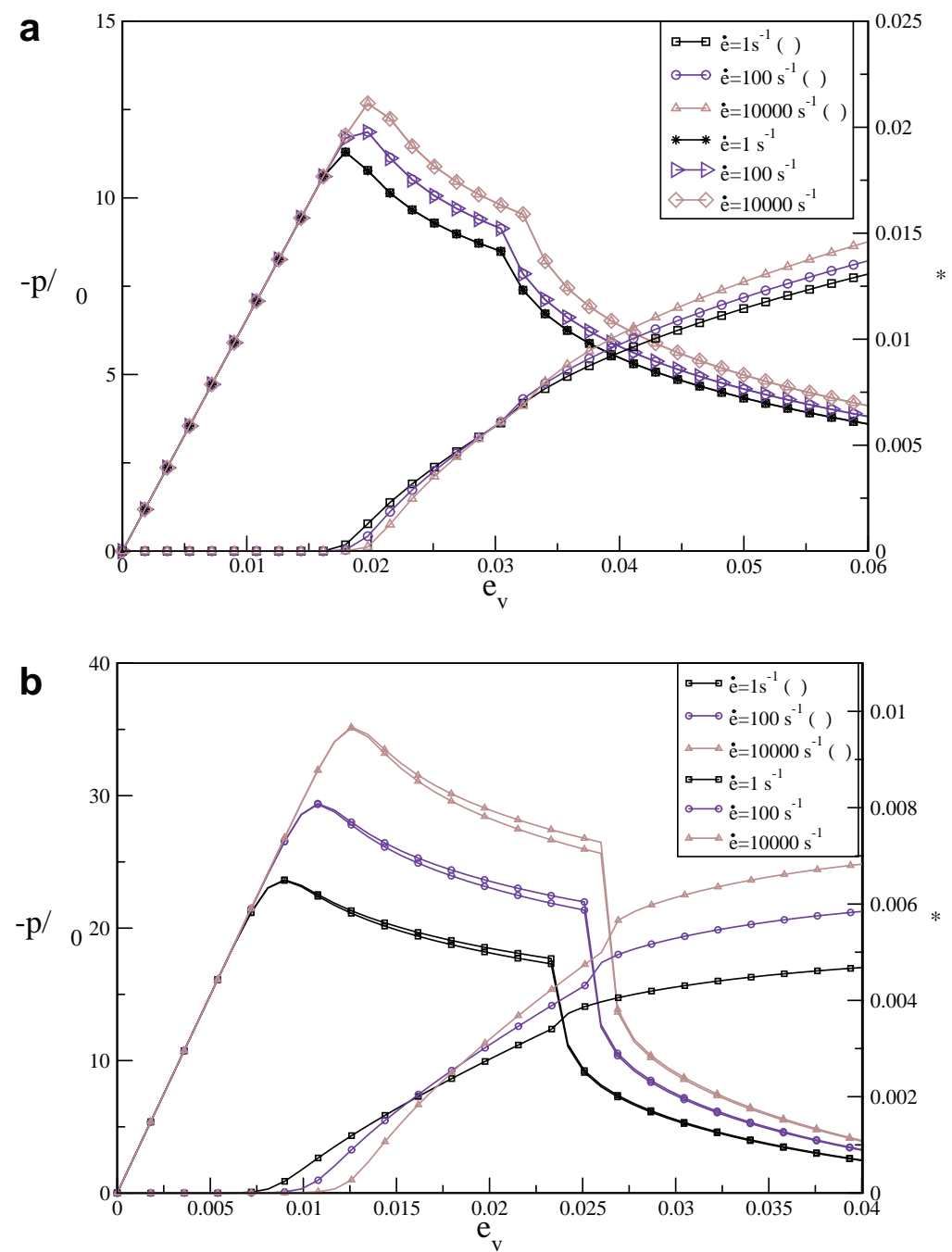

Fig. 3. Hydrostatic stress and homologous temperature as a function of volumetric strain. Volumetric expansion. (a) Material A. (b) Material B. ( $\theta$ ) indicates consideration of thermal effects. 
with

$\mathbf{s}^{\text {trial }}=\mathfrak{R}_{n+1} \mathbf{s}_{\mathfrak{R}}^{\text {trial }} \mathfrak{R}_{n+1}^{\mathrm{T}}$.

\section{Numerical results}

The performance of the proposed algorithm was proved by performing two kinds of analysis. Firstly, three singleelement cases have been considered with the algorithm implemented in the commercial finite element code ABAQUS/Standard [43] through the user subroutine UMAT. In this code, all tensors are relative to the Jaumann corotational basis. Secondly, different Taylor cylinder impact tests have been simulated, using in this case the subroutine $V U M A T$ that implements the proposed algorithm in the commercial code ABAQUS/Explicit [44]. In this code, all tensors are relative to the Green Naghdi McInnis corotational basis.

For calculations, the materials chosen were the ARMCO IRON, selected by Worswick and Pelletier [33], and the 4340 Steel, widely used in industry (for the single-element cases) and an Oxygen-free electronic copper for the Taylor cylinder impact test. All materials are modeled using the Johnson Cook flow equation [60] given by

$$
\sigma=\left(K_{1}+K_{2}\left(\varepsilon^{p}\right)^{n}\right)\left(1+K_{3} \ln \left(\max \left(1, \frac{\dot{\varepsilon}^{p}}{\dot{\varepsilon}_{0}}\right)\right)\right)\left(1-\theta^{* m}\right) .
$$

This equation considers the hardening effects of plastic strain, plastic strain rate and the softening effect of temperature in a multiplicative way. The first is defined through a Ludwig law with three constants $K_{1}, K_{2}$, and $n$. The strainrate effect is taken into account through a linear function with respect to the logarithm of the plastic strain rate, with a slope equal to $K_{3}$. The function "max" allows to define a lower limit of plastic strain-rate sensitivity, $\dot{\varepsilon}_{0}$. The parameter $m$ defines the temperature sensitivity, where $\theta^{*}$ is a homologous temperature defined as
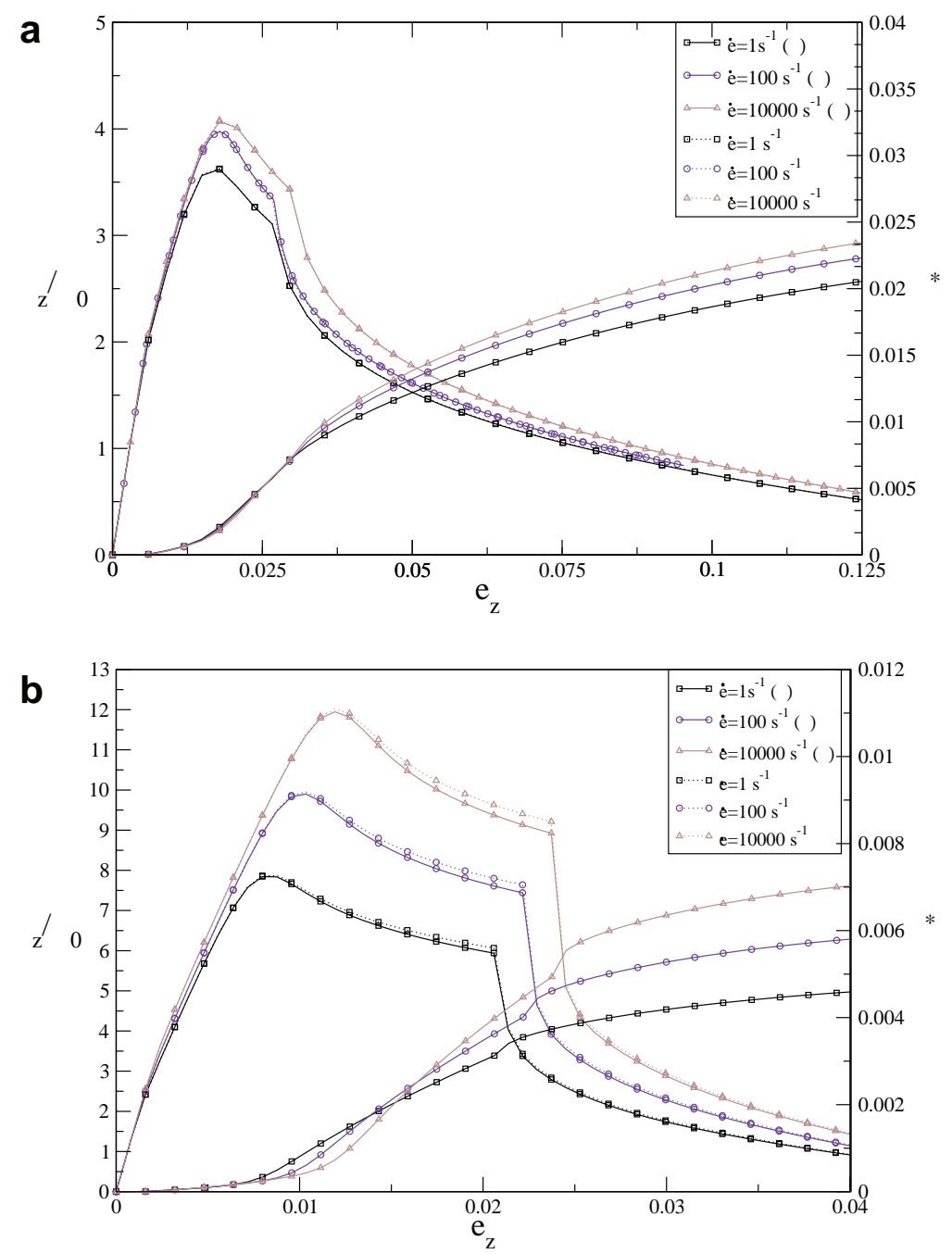

Fig. 4. Axial stress and homologous temperature as a function of axial strain. Constrained uniaxial extension. (a) Material A. (b) Material B. ( $(\theta)$ indicates consideration of thermal effects. 
$\theta^{*}=\frac{\theta-\theta_{0}}{\theta_{\mathrm{m}}-\theta_{0}}$

and $\theta_{0}$ and $\theta_{\mathrm{m}}$ the reference and melting temperatures, respectively.

The model proposed by Johnson and Cook is probably the most widely used among those accounting for plastic strain, plastic strain rate and temperature effects, since numerous efforts have been made in the past to determine their parameters for a large number of metallic materials.

The corresponding properties and parameters for the three materials, including the initial porosity $f_{0}$, are given in Tables 1 and 2.

\subsection{Single-element test cases}

The following three single-element test cases were analysed (see Fig. 1): (a) uniaxial extension, (b) volumetric expansion and (c) constrained uniaxial extension.
The three of them were previously chosen by Worswick and Pelletier [33] and Betegón et al. [36] in 2D plane-strain conditions and the first two were used by Aravas [55] in 2D plane-stress and 3D quasi-static conditions, respectively.

The initial element dimensions $l_{x}^{0}, l_{y}^{0}$ and $l_{z}^{0}$ were set equal to $1 \mathrm{~mm}$ and, for comparison, the axial engineering strain rate $\dot{e}_{z}=v_{z} / l_{z}^{0}$ used to run the cases took the values of $1,10^{2}$, and $10^{4} \mathrm{~s}^{2}$.

Figs. 24 show, for each load case, the evolution of a stress (axial stress in uniaxial extension and constrained uniaxial extension, hydrostatic stress in volumetric expansion) normalized by the static yield stress, as a function of a strain (axial strain in uniaxial extension and constrained uniaxial extension, logarithmic volumetric strain in volumetric expansion) for different strain rates and for both materials.

These results show that the strain rate increases both the stress level as well as the strain where the maximum stress
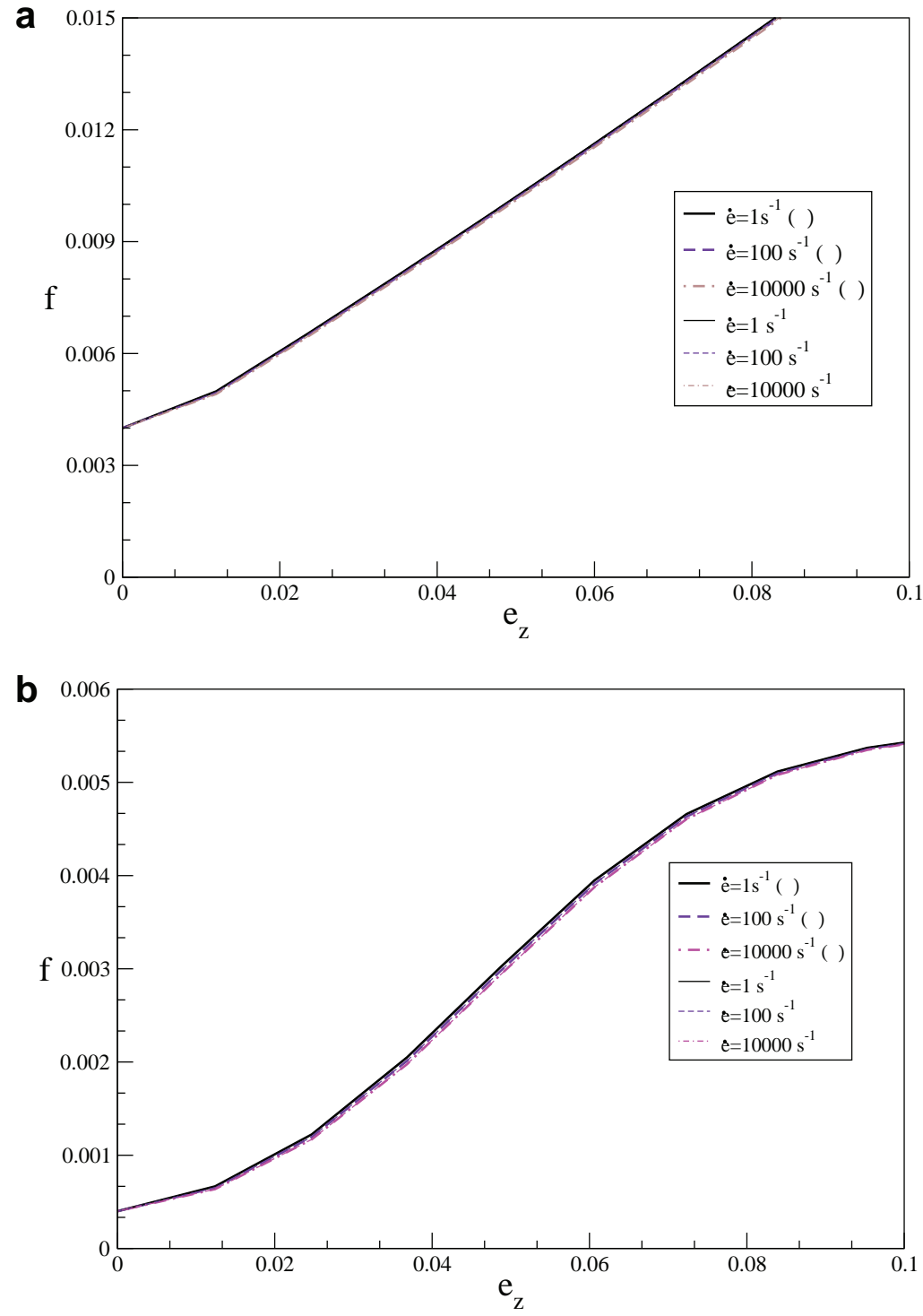

Fig. 5. Porosity as a function of axial strain. Uniaxial extension. (a) Material A. (b) Material B. ( $\theta$ ) indicates consideration of thermal effects. 
takes place. These findings are analogous with previous results $[33,36]$.

The softening effect of temperature can be observed especially in the first loading case (uniaxial extension) and Armco Iron (material B). The stronger effect in this case agrees with the increase in the homologous temperature. For the other loading cases the thermal effect is negligible compared to the strain-rate one.

The porosity evolution with the strain corresponding to the first loading case is given in Fig. 5 for the two materials considered and the different conditions studied. As can be seen, the porosity is practically unaffected by either the strain rate or the temperature.

Figs. 6 and 7 give the same information for the volumetric expansion case and uniaxial constrained case, respectively. These curves differ from those of the first case due to the high triaxiality developed in these configurations. However, the effect of temperature is negligible and the observed variation with the strain rate agrees with the results of Betegón et al. [36].

The convergence behaviour of the algorithm is shown in Table 3, which gives the maximum residual force in each iteration for the first time increment corresponding to the uniaxial extension case for a strain rate $\dot{e}_{z}=10^{4}$ and with consideration of thermal effects. It is worth noting that the tangent operator developed provides a quadratic rate of convergence.

\subsection{Taylor cylinder impact test}

To illustrate the performance of the developed algorithm in more complex loading configurations involving a large number of elements, seven different Taylor cylinder impact tests have been analysed. This test consists of launching a circular cylinder with an initial velocity against a rigid surface. During the impact, the large amount of
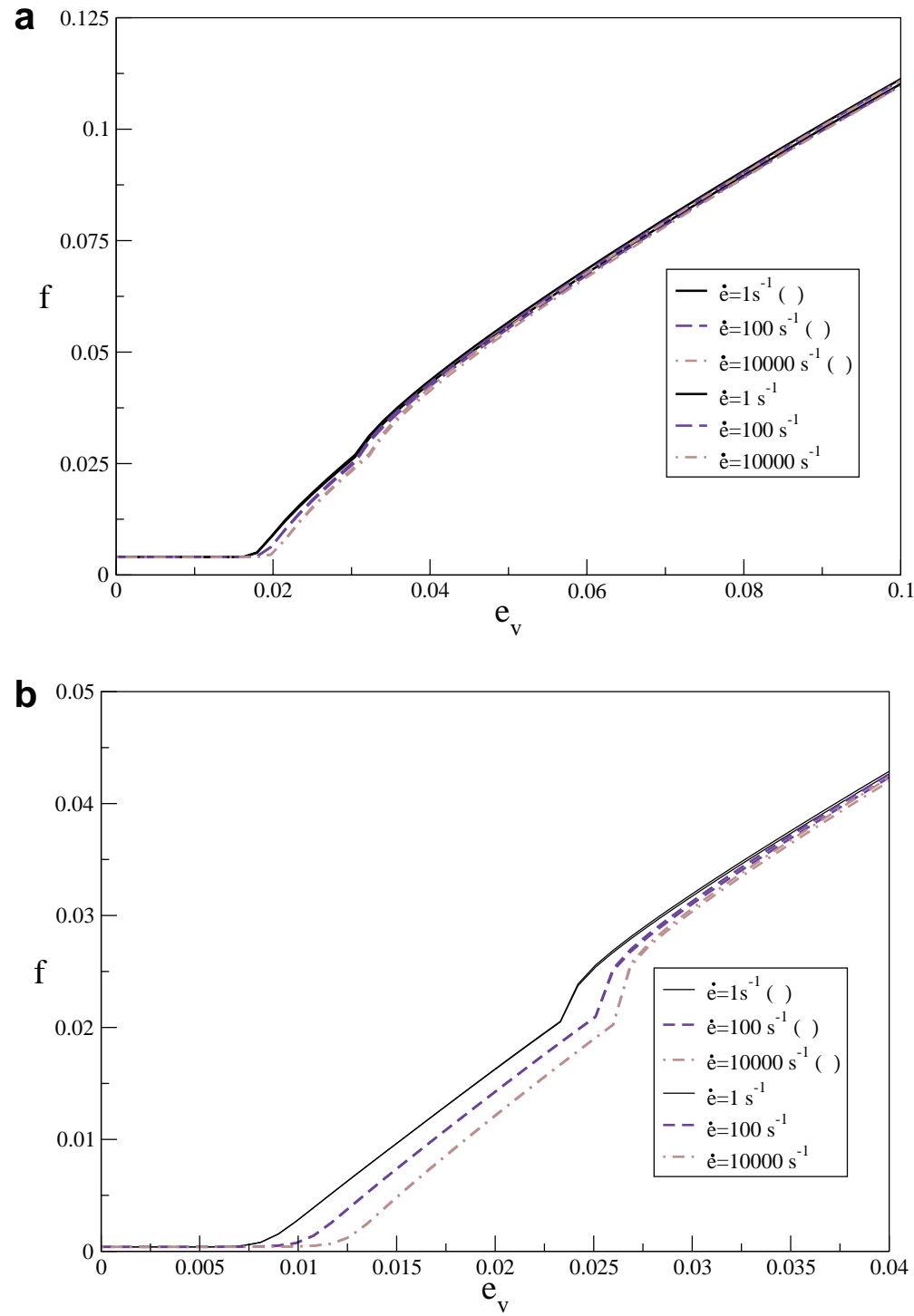

Fig. 6. Porosity as a function of volumetric strain. Volumetric expansion. (a) Material A. (b) Material B. ( $\theta$ ) indicates consideration of thermal effects. 

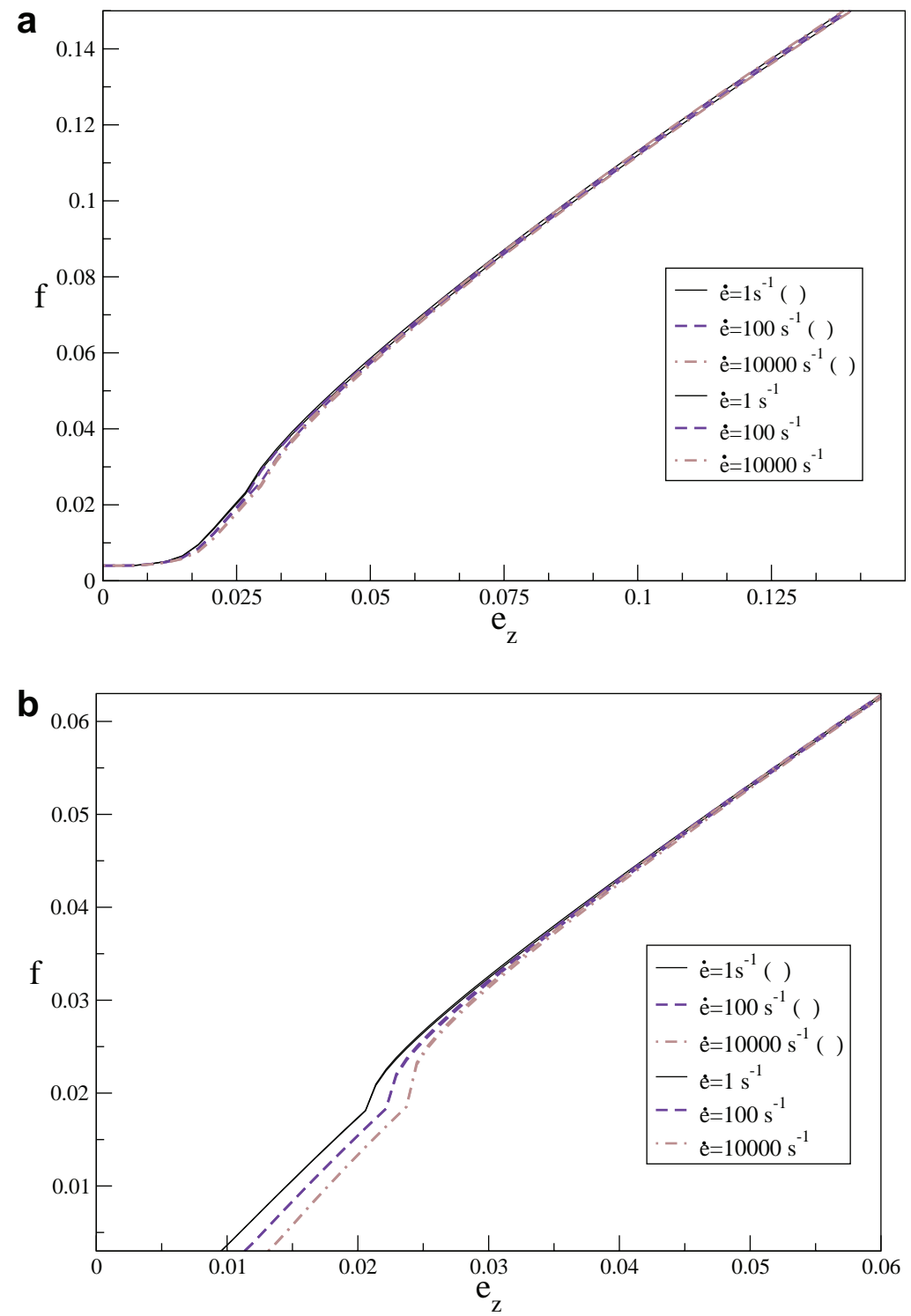

Fig. 7. Porosity as a function of axial strain. Constrained uniaxial extension. (a) Material A. (b) Material B. ( $\theta$ ) indicates consideration of thermal effects.

Table 3

Maximum residual force for the first time increment

\begin{tabular}{lll}
\hline & Material A (4340 Steel) & Material B (Armco Iron) \\
\hline Iteration 1 & 124 & 181 \\
Iteration 2 & 1.27 & 1.76 \\
Iteration 3 & $1.76 \mathrm{e} \mathrm{2}$ & $4.056 \mathrm{e} 2$ \\
Iteration 4 & $2.3 \mathrm{e} 4$ & $3.177 \mathrm{e} 4$
\end{tabular}

Cases of uniaxial extension and $\dot{e}_{z} \quad 10^{4} \mathrm{~s}^{-1}$ considering thermal effects.

plastic deformation in the specimen produces a shape that for the years has been used to estimate dynamic properties of the materials [61]. This kind of test has been analysed by Worwick and Pick [32], but without considering thermal effects.

The cylinder geometries and initial impact velocities selected (see Table 4) correspond to experiments performed by House [62] on oxygen-free electronic copper (see Tables 1 and 2 for the material properties).
The numerical models consist of 650, 1200, 1500, 1900 and 2500 four-node bilinear axisymmetric quadrilateral elements, with reduced integration and hourglass control for each of the five different specimens given in Table 4 (tests 15 , respectively). A mesh example is given in Fig. 8 corresponding to specimen number 1 . It can be observed that a fine mesh is generated in the vicinity of the impact face where large inelastic deformations are expected to occur, while a relatively coarse discretization is used in the rest of the cylinder where it is expected to remain nearly undeformed.

Table 4 shows the numerical and experimental values [62] of the ratio of final and initial radio of the cylinder $\left(R_{\mathrm{f}} / R_{\mathrm{i}}\right)$, and of the ratio of final and it initial length $\left(L_{\mathrm{f}} / L_{\mathrm{i}}\right)$ for the seven cases analysed. The error level, calculated as: error $=\frac{(\cdot)_{\text {exp }}-(\cdot) \text { num }}{(\cdot)_{\text {num }}}$, is in all cases less than $12 \%$. This value is acceptable, particularly if one considers that in the cylinders can undergo misalignment during impact [32]. 
Table 4

Taylor test results for oxygen free electronic copper

\begin{tabular}{|c|c|c|c|c|c|c|c|c|c|}
\hline \multirow[t]{2}{*}{ Simulated test } & \multirow[t]{2}{*}{ Initial diameter (mm) } & \multirow[t]{2}{*}{ Initial length (mm) } & \multirow[t]{2}{*}{ Impact velocity $(\mathrm{m} / \mathrm{s})$} & \multicolumn{3}{|l|}{$R_{\mathrm{f}} / R_{\mathrm{i}}$} & \multicolumn{3}{|l|}{$L_{\mathrm{f}} / L_{\mathrm{i}}$} \\
\hline & & & & Exp. & Sim. & Error $(\%)$ & Exp. & Sim. & Error $(\%)$ \\
\hline 1 & 7.595 & 11.39 & 191 & 1.58 & 1.76 & 11.4 & 0.78 & 0.70 & 10.3 \\
\hline 2 & 7.595 & 22.79 & 200 & 1.87 & 2.06 & 10.2 & 0.74 & 0.68 & 8.1 \\
\hline 3 & 7.595 & 30.38 & 175 & 1.74 & 1.86 & 6.9 & 0.78 & 0.72 & 7.7 \\
\hline 4 & 7.595 & 37.97 & 200 & 1.94 & 2.08 & 7.2 & 0.72 & 0.72 & 0 \\
\hline 5 & 7.595 & 56.96 & 153 & 1.55 & 1.61 & 3.9 & 0.83 & 0.75 & 9.6 \\
\hline 6 & 7.595 & 56.96 & 156 & 1.57 & 1.64 & 4.5 & 0.83 & 0.74 & 10.8 \\
\hline 7 & 7.595 & 56.96 & 189 & 1.94 & 1.91 & 1.5 & 0.73 & 0.66 & 9.6 \\
\hline
\end{tabular}

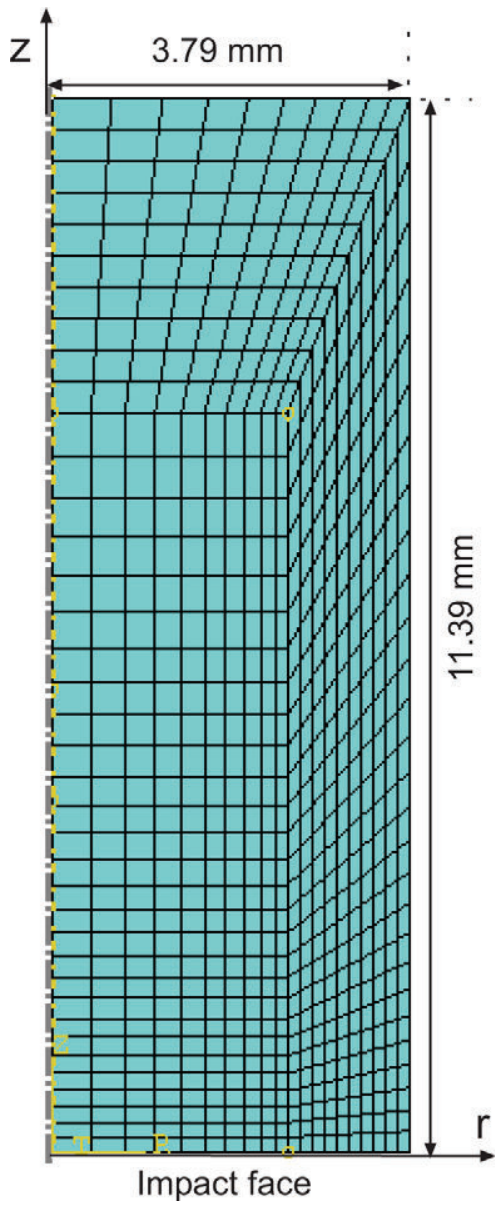

Fig. 8. Finite element mesh for the cylinder with initial geometry corresponding to specimen of test number 1 . The impact face is located at $z=0$.

Once numerical and experimental results have proved to be analogous, information concerning the evolution of several variables can be derived. Following the analysis made by Worwick and Pick [32] without consideration of thermal effects, the temporal evolution of hydrostatic stress and porosity in three elements has been determined. These elements are located on the cylinder axis at the impact face, at 2 and $4 \mathrm{~mm}$ away from the impact face, respectively. Moreover, the temperature evolution in these three elements has been computed.

The information given below corresponds to simulated test number 5 (see Table 4) with the initial cylinder length and diameter of 56.96 and $7.595 \mathrm{~mm}$, respectively, and initial impact velocity of $153 \mathrm{~m} / \mathrm{s}$.

Fig. 9 shows contours of the porosity, $f$, at 32 and $55 \mu$ s after impact, respectively. According to the results obtained by Teng et al. [63] for Taylor cylinder impact testing, damage by void growth is localized mainly in two zones of the specimen's tip: axis and periphery. The first one leads to the so-called confined-fracture in the Taylor specimen, which may generate a number of internal voids and even cracks inside the specimen although no cracks are visible on the surface. The second one may cause petalling at higher velocities due to the propagation of radial cracks towards the symmetry axis. The periphery of the cylinder nose moves backwards during mushrooming and the contact with the rigid wall is discontinued directly after impact. Then tensile hoop stresses predominate and the triaxiality becomes positive, leading to damage growth.

Fig. 10 plots the history of hydrostatic stresses, normalized by the yield stress, for the three elements on the axis. From 0 to $2.3 \mu \mathrm{s}$, the dimensionless hydrostatic stress is negative and reaches a maximum of 10.3 at $0.7 \mu \mathrm{s}$ in the impacting face. After that the contact with the rigid wall is discontinued for a few microseconds, these results being consistent with those of Worswick and Pick [32] and Teng et al. [63]. The hydrostatic stress changes to tension and this leads to void growth. During this period, several oscillations become visible at the element in the impacting face. These are due to radial unloading stress waves travelling round and round from the lateral surface to the axis [63]. As the gap closes, the compression becomes again dominant reaching a second maximum, and voids generated may initially close. This is consistent with the results shown in Fig. 11: in periods with negative hydrostatic stress (compressive stresses), there is a negative evolution of porosity; meanwhile, in periods corresponding to positive hydrostatic stress, an increase in the $f$ values occur. Similar behaviour was also found by Worswick and Pick [32]. The maximum value of the void-volume fraction is reached in the element located at $2 \mathrm{~mm}$ with a value of 0.011 at time $6 \mu$ s after impact.

Fig. 12 shows the evolution of homologous temperature, $\theta^{*}$, with time for the three aforementioned elements. In this case, a maximum value of 0.15 is reached for the element in the impact face at $45 \mu$ s after impact. Although plastic deformation continues until this moment, as can be 

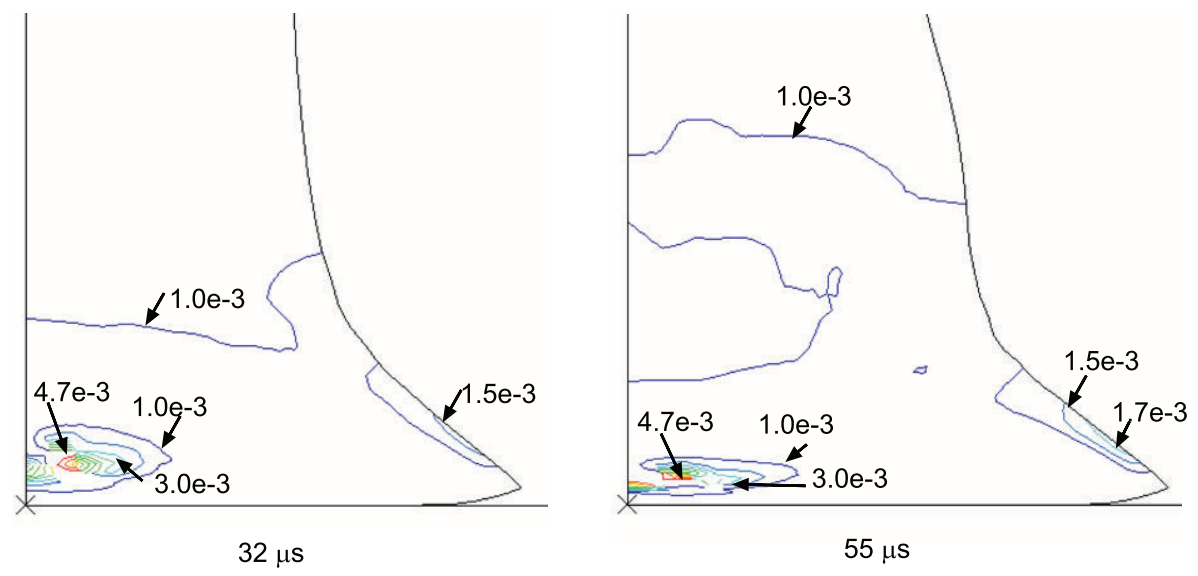

Fig. 9. Contours of porosity at 32 and $55 \mu$ s after impact. (Simulated test number 5.)

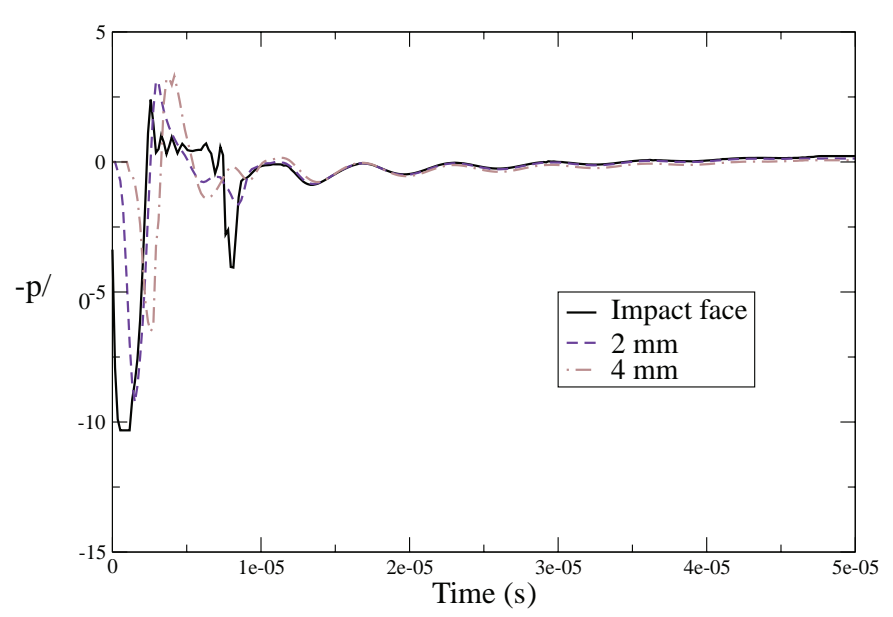

Fig. 10. Hydrostatic stress as a function of time for three elements on the specimen axis. (Simulated test number 5.)

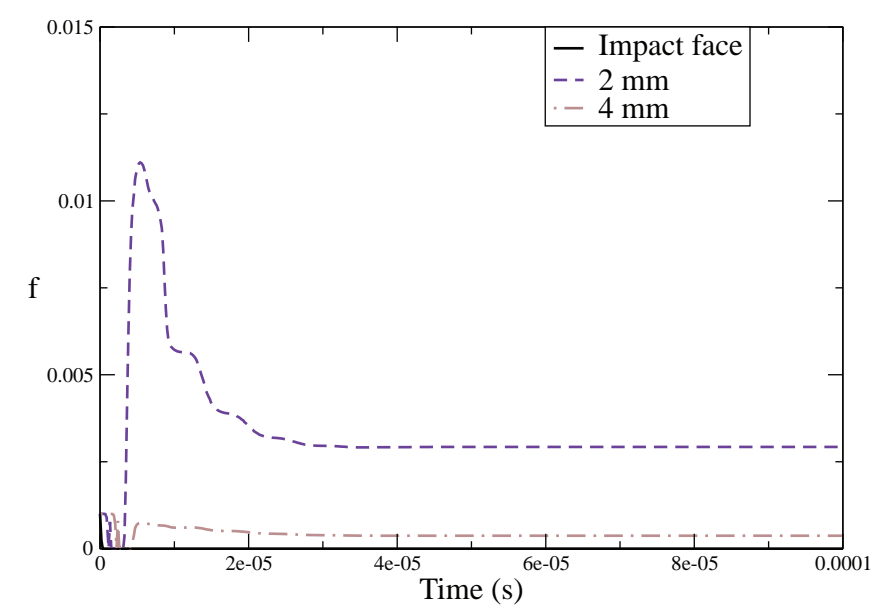

Fig. 11. Porosity as a function of time for three elements on the specimen axis. (Simulated test number 5.)

deduced from the temperature increase, the void growth stops long before.

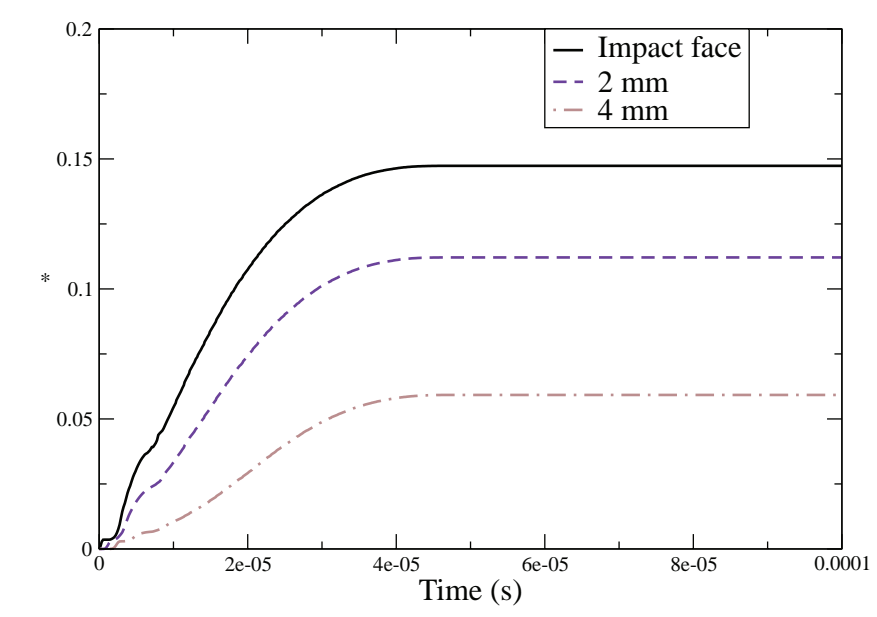

Fig. 12. Homologous temperature as a function of time for tree elements on the specimen axis. (Simulated test number 5.)

\section{Conclusions}

Reliable characterisations of materials at high strain rates and temperatures and precise modelling of ductile damage processes are necessary for the simulation of many advanced processes in engineering. This work presents a computational framework for finite strain thermoviscoplastic constitutive equations, coupled to the complete Gurson model. Following the consistent viscoplastic approach and using a fully implicit scheme, the discretized constitutive equations are presented in a corotational framework. The solution of these equations allows to update stress and state variables, and their differentiation leads to a closed form of the tangent operator. The method inherits the robustness and stability of classical return mapping algorithms, which were tested through the simulation of a single 3D element submitted to different load paths, as well as Taylor cylinder impact tests. These simulations also highlight the influence of the thermal effects on the softening of Gurson materials. 


\section{Acknowledgements}

This research was done with the financial support of the Spanish Ministry of Education under Project Reference DPI2005-06769, and of the Region of Madrid, under Project Reference UC3M-IME-05-054.

\section{References}

[1] J. Rojek, O.C. Zienkiewicz, E. Onate, E. Postek, Advanced in FE explicit formulation for simulation of metal forming processes, $\mathrm{J}$. Mater. Process. Technol. 119 (2001) 4147.

[2] A. Molinari, C. Musquar, G. Sutter, Adiabatic shear banding in high speed machining of $\mathrm{Ti} 6 \mathrm{Al} 4 \mathrm{~V}$ : experiments and modeling, Int. J. Plasticity 18 (2002) 443459.

[3] M. Baker, J. Rosler, C. Siemers, A finite element model of high speed metal cutting with adiabatic shearing, Comput. Struct. 80 (2002) 495 513.

[4] A. Reyes, M. Langseth, O.S. Hopperstad, Crashworthiness of aluminum extrusions subjected to oblique loading: experiments and numerical analyses, Int. J. Mech. Sci. 44 (2002) 19651984

[5] S. Yadav, E.A. Repetto, G. Ravichandran, M. Ortiz, A computational study of the influence of thermal softening on ballistic penetration in metals, Int. J. Impact Engrg. 25 (2001) 787803.

[6] Z. Rosenberg, E. Dekel, On the role of material properties in the terminal ballistics of long rods, Int. J. Impact Engrg. 30 (2004) 835 851.

[7] P. Perzyna, Fundamental problems in viscoplasticity, Advances in Applied Mechanics, vol. 9, Academic Press, New York, 1966, pp. 243277.

[8] G. Duvaut, J.L. Lions, Les Inequations en Mechanique et en Physique, Dunod, Paris, 1972.

[9] W.M. Wang, L.J. Sluys, R. de Borst, Viscoplasticity for instabilities due to strain softening and strain rate softening, Int. J. Numer. Method Engrg. 40 (1997) 38393864.

[10] M. Ristinmaa, N.S. Ottosen, Consequences of dynamic yield surface in viscoplasticity, Int. J. Solid Struct. 37 (2000) 46014622.

[11] A. Winnicki, C.J. Pearce, N. Bićanić, Viscoplastic Hoffman consis tency model for concrete, Comput. Struct. 79 (2001) 719.

[12] O.M. Heeres, Modern strategies for the numerical modelling of the cyclic and transient behaviour of soils, Ph.D. Thesis, Tech. Univ. Delft, 2001.

[13] O.M. Heeres, A.S.J. Suiker, R. de Borst, A comparison between the Perzyna viscoplastic model and the Consistency viscoplastic model, Eur. J. Mech. A: Solid 21 (2002) 112.

[14] A. Molinari, Collective behavior and spacing of adiabatic shear bands, J. Mech. Phys. Solid 45 (1997) 15511575.

[15] N. Charalambakis, T. Baxevanis, Adiabatic shearing of non homo geneous thermoviscoplastic materials, Int. J. Plasticity 20 (2004) 899 914.

[16] A. Rusinek, R. Zaera, J.R. Klepaczko, R. Cheriguene, Analysis of inertia and scale effects on dynamic neck formation during tension of sheet steel, Acta Mater. 53 (2005) 53875400.

[17] F. Zhou, T.W. Wright, K.T. Ramesh, The formation of multiple adiabatic shear bands, J. Mech. Phys. Solid 54 (2006) 13761400.

[18] J.P. Ponthot, Unified stress update algorithms for the numerical simulation of large deformation elasto plastic and elasto viscoplastic processes, Int. J. Plasticity 18 (2002) 91126.

[19] L. Mahler, M. Ekh, K. Runesson, A class of thermo hyperelastic viscoplastic models for porous materials: theory and numerics, Int. J. Plasticity 17 (2001) 943969

[20] J.R. Zaera, J. Fernandez Saez, An implicit consistent algorithm for the integration of thermoviscoplastic constitutive equations in adia batic conditions and finite deformations, Int. J. Solid Struct. 43 (2006) 15941612
[21] A.L. Gurson, Continuum theory of ductile rupture by void nucleation and growth part I. Yield criteria and flow rules for porous ductile media, J. Engrg. Mater. Technol. 99 (1977) 215.

[22] V. Tvergaard, Influence of voids on shear band instabilities under plane strain conditions, Int. J. Fract. 17 (1981) 389407.

[23] V. Tvergaard, On localization in ductile materials containing spher ical voids, Int. J. Fract. 18 (1982) 237252.

[24] V. Tvergaard, A. Needleman, Analysis of cup cone fracture in a round tensile bar, Acta Metall. 32 (1984) 157169.

[25] P.F. Thomason, Ductile fracture of metals, Pergamon Press, Oxford, 1990.

[26] Z.L. Zhang, A complete Gurson Model, in: M.H. Aliabadi. Nonlin ear Fracture and Damage Mechanics, WIT, Southampton, 2001, pp. 223248.

[27] Z.L. Zhang, C. Thaulow, J. Ødegård, A complete Gurson model approach for ductile fracture, Engrg. Fract. Mech. 67 (2000) 155168.

[28] T. Pardoen, J.W. Hutchinson, An extended model for void growth and coalescence, J. Mech. Phys. Solid 48 (2000) 24672512.

[29] J. Pan, M. Saje, A. Needleman, Localization of deformation in rate sensitive porous plastic solids, Int. J. Fract. 21 (1983) 261278.

[30] D. Pierce, C.F. Shih, A. Needleman, A tangent modulus method for rate dependent solids, Comput. Struct. 18 (1984) 875887.

[31] V. Tvergaard, A. Needleman, Effect of material sensitivity on failure modes in the Charpy V Notch test, J. Mech. Phys. Solid 34 (1986) 213241.

[32] M.J. Worswick, R.J. Pick, Void growth and coalescence during high velocity impact, Mech. Mater. 19 (1995) 293309

[33] M.J. Worswick, P. Pelletier, Numerical simulation of ductile fracture during high strain rate deformation, Eur. Phys. J. 42 (2005) 2929 2948.

[34] R. Becker, Ring fragmentation predictions using the Gurson model with material stability conditions as failure criteria, Int. J. Solid Struct. 39 (2002) 35553580.

[35] T. Børvik, O.S. Hopperstad, T. Berstad, On the influence of stress triaxiality and strain rate on the behaviour of a structural steel. Part II. Numerical study, Eur. J. Mech. A: Solid 22 (2003) 1532.

[36] C. Betegón, J.J. del Coz, I. Penuelas, Implicit integration procedure for viscoplastic Gurson materials, Comput. Method Appl. Mech. Engrg. 195 (2006) 61466157.

[37] A. Srikanth, N. Zabaras, A computational model for the finite element analysis of thermoplasticity coupled with ductile damage at finite strains, Int. J. Numer. Method Engrg. 45 (1999) 15691605.

[38] K.C. Koppenhoefer, R.H. Dodds, Ductile crack growth in pre cracked CVN specimens: numerical studies, Nucl. Engrg. Des. 180 (1998) 221241.

[39] A. Eberle, D. Klingbeil, J. Schicker, The calculation of dynamic JR curves from the finite element analysis of a charpy test using a rate dependent damage model, Nucl. Engrg. Des. 198 (2000) 7587.

[40] A. Needleman, V. Tvergaard, A numerical study of void distribution effects on dynamic, ductile crack growth, Engrg. Fract. Mech. 38 (1991) 157173

[41] V. Tvergaard, A. Needleman, Analysis of the Charpy V notch test for welds, Engrg. Fract. Mech. 65 (2000) 627643.

[42] S. Hao, W. Brocks, The Gurson Tvergaard Needleman model for rate and temperature dependent materials with isotropic and kine matic hardening, Comput. Mech. 20 (1997) 3440.

[43] ABAQUS/Standard v6.5 User's Manual, ABAQUS Inc., Richmond, USA, 2005.

[44] ABAQUS/Explicit v6.5 User's Manual, ABAQUS Inc., Richmond, USA, 2005

[45] E. Kroner, Allgemeine kontinuumstheorie der versetzungen und eigen spannungen, Arch. Rational Mech. Anal. 4 (1959) 273334.

[46] E.H. Lee, Elastic plastic deformation at finite strains, J. Appl. Mech. 36 (1969) 16.

[47] S. Nemat Nasser, On finite deformation elasto plasticity, Int. J. Solid Struct. 18 (1982) 857872.

[48] A. Kahn, Continuum Theory of Plasticity, John Wiley and Sons, New York, 1995. 
[49] A. Needleman, J.R. Rice, Limits to ductility set by plastic flow localization, in: D.P. Koistinen, N.M. Wang (Eds.), Mechanics of Sheet Metal Forming, Plenum Press, New York, 1978, pp. 237267.

[50] C.C. Chu, A. Needleman, Void nucleation effects in biaxially stretched sheets, J. Engrg. Mater. Technol. 102 (1980) 249256.

[51] J.C. Simo, T.J.R. Hughes, Computational Inelasticity, Springer, New York, 1998.

[52] I. Doghri, Mechanics of deformable solids: linear and nonlinear, analytical and computational aspects, Springer, Berlin, 2000.

[53] B. Hagege, Simulation du comportement mécanique des milieux fibreux en grandes transformations: application aux renforts tricotés, Ph.D. Thesis, ENSAM, Paris, 2004.

[54] T.J.R. Hughes, J. Winget, Finite rotation effects in numerical integration of rate constitutive equations arising in large deformation analysis, Int. J. Numer. Method Engrg. 15 (1980) 18621867.

[55] N. Aravas, On the numerical integration of a class of pressure dependent plasticity models, Int. J. Numer. Method Engrg. 24 (1987) 13951416.

[56] J.C. Simo, R.L. Taylor, Consistent tangent operators for rate independent elastoplasticity, Comput. Method Appl. Mech. Engrg. 48 (1985) 101118.
[57] Z.L. Zhang, On the accuracies of numerical integration algorithms for Gurson based pressure dependent elastoplastic constitutive mod els, Comput. Method Appl. Mech. Engrg. 121 (1995) 1528.

[58] R.C. Lin, W. Brocks, On a finite strain viscoplastic theory based on a new internal dissipation inequality, Int. J. Plasticity 20 (2004) 1281 1311.

[59] R.C. Lin, W. Brocks, J. Betten, On internal dissipation inequalities and finite strain inelastic constitutive laws: theoretical and numerical comparisons, Int. J. Plasticity 22 (2006) 18251857.

[60] G.R. Johnson, W.H. Cook, A constitutive model and data for metals subjected to large strains, high strain rates, and temperatures, in: Proceedings of 7th International Symposium Ballistics, The Hague, The Netherlands, 1983, pp. 17.

[61] W.K. Rule, A numerical scheme for extracting strength model coefficients from Taylor test data, Int. J. Impact Engrg. 19 (9 10) (1997) 797810.

[62] J.W. House, Tech. Rep. AFATL TR 89 41, Air Force Armament Laboratory, Eglin AFB, FL, 1989.

[63] X. Teng, T. Wierzbicki, S. Hiermaier, I. Rohr, Numerical prediction of fracture in the Taylor test, Int. J. Solid Struct. AP 4 (1998) 257 267. 